\title{
Good modeling of bad outputs: editors' introduction
}

\author{
Subal C. Kumbhakar ${ }^{1}$ • Emir Malikov ${ }^{2}$
}

Published online: 27 March 2017

(C) Springer-Verlag Berlin Heidelberg 2017

This special issue of Empirical Economics brings together researchers actively working in the field of production analysis to discuss present challenges (and solutions thereto) associated with the proper modeling of production processes in the presence of bad outputs. The objective of this special issue is to contribute to the emerging literature on robust economic models and econometric methods suitable for the analysis of production technologies when one or more outputs are economically and/or socially undesirable, or so-to-say "bad." The special issue presents a strong set of papers, each of which either offers novel theoretical conceptualization or econometric methodology related to modeling production processes with bad outputs or else applies recent methodological developments in new contexts or both.

The by-production of bad outputs is an inherent attribute of many production processes. In agriculture, some examples of such processes include the use of pesticides in farming which, while increasing crop yields (good outputs), also results in an increase in undesirable and often non-marketed environmental risks associated with leaching and runoff, or dairy farming where the production of desirable dairy products like milk is accompanied by the natural but undesirable generation of nitrogen surpluses. Moreover, the by-production of bad outputs is not confined to agricultural production only. The concept of bad outputs can also be extended to carbon-based electric power generation that is accompanied by the emission of pollutant gases or even to the provi-

Subal C. Kumbhakar

kkar@binghamton.edu

Emir Malikov

emalikov@auburn.edu

1 Department of Economics, State University of New York at Binghamton, Binghamton, NY, USA

2 Department of Agricultural Economics and Rural Sociology, Auburn University, Auburn, AL, USA 
sion of banking services which suffers from the by-production of undesirable outputs like non-performing loans due to banks' inherent exposure to credit risk.

The aforementioned examples highlight the importance of accounting for bad outputs when modeling firms' production processes. One ought not to disregard bad outputs. Not only does the failure to properly accommodate the presence of bad outputs preclude researchers from crediting firms' productivity and efficiency for a ceteris paribus reduction in undesirable outputs (pollutants), but it also jeopardizes the reliability of the estimates of a desirable production process. Both aspects are rather crucial from a policy perspective, since both technical and environmental efficiency/productivity along with other technological metrics are often taken as the basis for devising new or evaluating already existing industry regulations.

Based on their approach to modeling bad outputs, papers included in this issue can loosely be put into two categories. The first group of papers considers a multi-equation representation of polluting technology, while the second group adopts an alternative single-equation specification of the production process in the presence of bad outputs. The multi-equation representation primarily attributed to Fernández et al. (2002, 2005), Forsund (2009) and Murty et al. (2012) relies on the more traditional multiplicative radial formulation of a system of a desirable technology and its accompanying undesirable by-production, whereas the single-equation approach usually formalizes polluting technology in the form of a single additive directional distance function under the joint weak disposability of good and bad outputs in the spirit of Chambers et al. (1996), Chung et al. (1997) and Färe et al. (2005). These two alternative approaches are arguably the most prevalent among practitioners in the literature. ${ }^{1}$ Interestingly, our special issue also includes papers that study a single-equation model of polluting technology based on a multiplicative radial distance function adapted to meaningfully accommodate the presence of bad outputs. In what follows, we briefly summarize the papers contributed to the special issue with articles considering a multi-equation framework being discussed first.

Murty and Russell study the link between two recent approaches to modeling emission-generating technologies: the by-production approach and the axiomatic approach. The by-production approach models these technologies as intersections of two independent sub-technologies reflecting, respectively, the relations between goods in intended output production designed by human engineers and the emissiongenerating mechanism of nature governed by material balance considerations. The axiomatic approach proposes a set of axioms that a pollution-generating technology should satisfy. The authors show that the by-production technology satisfies these axioms and that, conversely, any technology satisfying the axioms can be decomposed into two sub-technologies satisfying the by-production properties. In either approach, the technology can be functionally represented by two radial distance functions with well-defined properties. These distance functions can also serve as measures of technological and environmental efficiency. Murty and Russell then exploit the link between the by-production and axiomatic approaches to offer preliminary suggestions about suitable functional forms for the empirical estimation of the two distance functions.

\footnotetext{
1 For an excellent review of different approaches to modeling bad outputs, also see Dakpo et al. (2016).
} 
In their paper, Ray, Mukherjee and Venkatesh adopt the aforementioned byproduction interpretation of bad output generation and conceptualize the good output as technologically separable from the bad output. However, the authors propose modeling a bad output-generating sub-technology in a novel manner. First, Ray et al. assume joint disposability of the bad output and the polluting input in the relevant sub-technology implying that the bad output can only be reduced if the polluting input is reduced as well. Second, they use the hyperbolic rendition of the directional distance function where the good output is increased while the bad output (along with the polluting input) is reduced by the same proportion. They then set up an integrated DEA optimization problem over the intersection of the two sub-technologies to measure the efficiency of a polluting firm. Notably, Ray et al. use the same intensity vectors in constructing the reference input-output bundles in the good and bad production technologies which, the authors argue, ensures a single peer group for any inefficient unit. In an empirical illustration of their methodology, the authors use aggregate data for 64 countries in the 1986-2011 period where per capita GDP is the good and per capita $\mathrm{CO}_{2}$ emission is the bad output. They utilize the results to compute opportunity costs of a targeted reduction in $\mathrm{CO}_{2}$ emission in terms of required dollar amounts of reduction in per capita GDP for individual countries in selected years.

The key feature of modeling joint production of intended/good outputs and unintended residuals (bads) is that the latter stem from the use of material inputs. Building on the factorially determined multi-output model of classical production theory, Førsund develops a multi-equation model which satisfies the so-called materials balance condition according to which the mass contained in inputs cannot disappear but must turn up in the desirable outputs or end up as undesirable residuals. In his model, Førsund is able to simplify complex technological relationships by expressing each of the good and bad outputs as functions of the same set of inputs. The proposed model is further augmented to incorporate end-of-pipe abatement activities. The author then derives the optimal planning solution using the concept of an environmental damage function for the impact of discharge of residuals into the natural environment. Førsund also shows that the traditional environmental policy instruments like direct regulation restricting the amount of undesirable residuals discharged into the environment, a Pigou tax on pollutants and cap and trade all function well. He then extends his model to allow for technical inefficiency and introduces three efficiency measures: desirable output efficiency, bad residual efficiency and abatement efficiency.

We next briefly overview the papers that build on a single-equation model of production.

From a theoretical point of view, most single-equation models of production technology with bad outputs fall into two broad types. The first type of models transforms the data on bad outputs in order to incorporate the transformed values as good outputs onto which the standard Shephard's axioms of production are then imposed. In this case, one usually may continue using the traditional radial distance functions to represent the technology. In contrast, the second type of models extends Shephard's disposability axioms and incorporates the original data on bads using the newly modified axioms. In most instances, researcher then adopts directional distance functions to formulate the polluting technology. Rather surprisingly, while the advantages/disadvantages of these competing models have been discussed quite 
extensively from a production theoretical point of view, however, very little empirical evidence regarding the consequences of these different approaches for applied research is available. In his paper, Hampf seeks to fill this void by comparing how alternative disposability assumptions about bad outputs affect the measurement of technological efficiency in practice. He quantifies and analyzes differences among competing theoretical models of production using data on electricity generation in the USA in 2011. In addition, Hampf also conducts extensive Monte Carlo simulations to evaluate the ability of the models to correctly estimate inefficiency in different specifications of the pollution-generating process.

Bostian, Färe, Grosskopf, Lundgren and Weber extend recent advances in time substitution modeling to a directional distance function framework, in order to examine the environmental performance of firms in Sweden's pulp and paper industry in 20022008. Their data allow them to estimate the optimal reallocation of environmental investments, expenditures and energy use to simultaneously maximize (desirable) production output and minimize emissions (bad output) in the years immediately before and after the implementation of the European Union Emissions Trading Scheme. The authors find evidence of overall productivity decline when considering both emissions and output objectives, due primarily to technological decline. Their results also suggest that cumulative dynamic inefficiency outweighs static inefficiency. Based on a comparison of optimal investment with the observed investment, Bostian et al. conclude that firms could have improved their performance by reallocating environmental investments to early periods and production-oriented investment to later periods.

In their paper, Pham and Zelenyuk contribute to the literature by generalizing the slack-based directional distance function introduced by Färe and Grosskopf (2010) to measure efficiency in the presence of bad outputs. They also accordingly adapt the revenue efficiency decomposition. To illustrate their model, the authors apply it to data on Vietnamese commercial banks during the 2008-2014 period. Pham and Zelenyuk compare results from their slack-based directional distance function to those based on the more standard directional distance function, the "enhanced" hyperbolic efficiency measure and the Farrell-type technical efficiency and find that the former has greater discriminative power.

Chambers and Serra extend the conventional technical efficiency analysis to a consideration of both environmental and social issues. They do so by incorporating the firm's corporate social responsibility activities (e.g., pollution abatement, improvement of worker health, worker training) into its production technology via treating the former as netputs to production. Essentially, the authors propose a DEA model to quantify the social dimension of firm performance. Production of good outputs and bad by-products such as $\mathrm{CO}_{2}$ emissions and the number of worker injuries/fatalities is combined in the measurement of firm's overall performance. Chambers and Serra showcase their model using a sample of global firms from Thompson Reuters's data stream and conclude that these firms exhibit strikingly low social performance levels.

The next two papers address issues concerning proper econometric modeling of directional distance functions in the presence of bad outputs.

When estimating a directional distance function, researchers typically make three restrictive assumptions. Specifically, they tend to assume (i) a pre-specified direction of movement of firm production toward the frontier in order to measure inefficiency, 
(ii) exogeneity of all inputs and outputs except for the normalized one and (iii) allocative efficiency of actual quantities of inputs and outputs. Atkinson and Tsionas's paper aims to relax each of these assumptions. The authors do not assume but estimate optimal directions which correspond to the firm's profit-maximizing position. They also generalize the directional distance function to a "shadow-quantity" directional distance function, which entails adding distortion parameters to each input and output quantity thereby creating shadow quantities. These quantities are price efficient subject to profit maximization based on market prices. To estimate the shadow quantities along with structural parameters, Atkinson and Tsionas form a system, which includes the shadow directional distance function and the first-order price equations from the shadow profit maximization problem. To accommodate potential endogeneity of inputs and outputs, they suggest employing prices of good inputs and good outputs as instruments and estimate the system via GMM.

Feng, Wang and Serletis's paper is also concerned with making the estimated directional distance function more flexible and thus robust. The authors estimate the shadow prices of $\mathrm{CO}_{2}$ emissions of electric utilities in the USA during 2001-2014 using a random coefficient, random directional vector directional output distance function model. The main feature of their proposed model is that both its coefficients and directional vector are allowed to vary across firms thus allowing different firms to have different production technologies and to follow different growth paths. Based on the Bayes factor analysis, Feng et al. find that their flexible model is strongly favored over the commonly used fixed coefficient counterpart. Their results suggest that the firmspecific average shadow prices differ significantly across electric utilities as well as show an upward trend.

While also pursing a single-equation approach to modeling polling technology, the last two articles in this special issue, however, do not rely on the additive directional distance function but rather seek to meaningfully adapt Shephard's multiplicative radial distance function to the presence of bad outputs. For instance, Färe, Grosskopf, Pasurka and Shadbegian specify a radial input distance function to model the joint production of good and bad outputs, based on which they investigate the employment effects of environmental regulations. While Morgenstern et al. (2002) are already well known for a similar investigation, they estimate a cost function representation of the polluting technology which is, however, significantly handicapped by its reliance on survey data of the costs of inputs assigned to pollution abatement. By contrast, Färe et al. can measure the relative importance of factors associated with changes in employment without (noisy) pollution abatement cost data. The authors operationalize their model using a sample of 80 coal-fired electric power plants operating in the USA during 1995-2005.

Despite some recent criticisms, the conventional radial distance function, which treats undesirable by-products as either frontier shifters or inputs, remains a popular go-to formulation of polluting production processes among practitioners. This unfading popularity is arguably driven by the ability of radial distance functions, unlike alternative directional distance functions, to allow for unit-free multiplicative changes in arguments as well as, by implicitly postulating the radial direction, to free researchers from the dilemma of having to explicitly choose the directional vector. In their paper, Malikov, Bokusheva and Kumbhakar therefore offer a generalization of the standard 
radial distance function to polluting technologies that can accommodate undesirable by-products in a more economically meaningful way. Specifically, they propose modeling undesirable outputs via a hedonic output index, which is meant to ensure that pollutants are treated as outputs, as opposed to inputs or theoretically unregulated frontier shifters, while also recognizing their undesirable nature. By using a radial input distance function generalized to encompass an (unobservable) hedonic output index of desirable and undesirable outputs, Malikov et al. are able to meaningfully describe relationships between different products (including the complementarity of desirable and undesirable outputs) within producible output sets as well as to represent technically feasible polluting production possibilities given inputs. An empirical application of their methodology to the case of Dutch dairy farms in 2001-2009 demonstrates the complexity of interactions between outputs, thereby attesting to the value of more elaborate representations of production possibilities.

On a final note, all of the papers selected for this special issue have gone through the usual process of peer review for Empirical Economics. We were fortunate to have an army of capable referees who provided excellent criticism and feedback on the papers in this issue. We would like to personally thank them for their dedicated service in helping us put this special issue together and ensuring that the submissions were of sufficient quality for publication. (Our own paper included in the issue was handled independently by a different editor of the journal.) We also owe a great debt of appreciation to the editorial board of Empirical Economics for approving the project. Lastly but not least importantly, we would like to thank Liane Wolf for impeccable behind-the-scenes assistance at various stages in the preparation of this issue.

\section{References}

Chambers RG, Chung Y, Färe R (1996) Benefit and distance functions. J Econ Theory 70:407-419

Chung Y, Färe R, Grosskopf S (1997) Productivity and undesirable outputs: a directional distance function approach. J Environ Manag 51:229-240

Dakpo KH, Jeanneaux P, Latruffe L (2016) Modelling polluting-generating technologies in performance benchmarking: recent developments, limits and future prospects in the nonparametric framework. Eur J Oper Res 250:347-359

Färe R, Grosskopf S (2010) Directional distance functions and slack-based measures of efficiency. Eur J Oper Res 200:320-322

Färe R, Grosskopf S, Noh D-W, Weber W (2005) Characteristics of a polluting technology: theory and practice. J Econom 126:469-492

Fernández C, Koop G, Steel MFJ (2002) Multiple-output production with undesirable outputs: an application to nitrogen surplus in agriculture. J Am Stat Assoc 97(458):432-442

Fernández C, Koop G, Steel MFJ (2005) Alternative efficiency measures for multiple-output production. J Econom 126:411-444

Forsund F (2009) Good modelling of bad outputs: pollution and multiple-output production. Int Rev Environ Resour Econ 3:1-38

Morgenstern RD, Pizer WA, Shih J-S (2002) Jobs versus the environment: an industry-level perspective. J Environ Econ Manag 43:412-436

Murty S, Russell RR, Levkoff SB (2012) On modeling pollution-generating technologies. J Environ Econ Manag 64:117-135 